\title{
Statistics of the largest geomagnetic storms per solar cycle (1844-1993)
}

\author{
D. M. Willis ${ }^{1, *}$, P. R. Stevens ${ }^{1,2}$, S. R. Crothers ${ }^{1}$ \\ ${ }^{1}$ Rutherford Appleton Laboratory, Chilton, Didcot, Oxon OX11 0QX, UK \\ ${ }^{2}$ Holmes Chapel Comprehensive School, Holmes Chapel, Crewe, Cheshire CW4 7DX, UK
}

Received: 8 January 1996 / Revised: 1 December 1996 / Accepted: 20 January 1997

\begin{abstract}
A previous application of extreme-value statistics to the first, second and third largest geomagnetic storms per solar cycle for nine solar cycles is extended to fourteen solar cycles (1844-1993). The intensity of a geomagnetic storm is measured by the magnitude of the daily $a a$ index, rather than the half-daily $a a$ index used previously. Values of the conventional $a a$ index (18681993), supplemented by the Helsinki $A k$ index (18441880), provide an almost continuous, and largely homogeneous, daily measure of geomagnetic activity over an interval of 150 years. As in the earlier investigation, analytic expressions giving the probabilities of the three greatest storms (extreme values) per solar cycle, as continuous functions of storm magnitude $(a a)$, are obtained by least-squares fitting of the observations to the appropriate theoretical extreme-value probability functions. These expressions are used to obtain the statistical characteristics of the extreme values; namely, the mode, median, mean, standard deviation and relative dispersion. Since the $A k$ index may not provide an entirely homogeneous extension of the $a a$ index, the statistical analysis is performed separately for twelve solar cycles (1868-1993), as well as nine solar cycles (1868-1967). The results are utilized to determine the expected ranges of the extreme values as a function of the number of solar cycles. For fourteen solar cycles, the expected ranges of the daily $a$ index for the first, second and third largest geomagnetic storms per solar cycle decrease monotonically in magnitude, contrary to the situation for the half-daily $a a$ index over nine solar cycles. The observed range of the first extreme daily $a a$ index for fourteen solar cycles is 159-352 nT and for twelve solar cycles is 215-352 nT. In a group of 100 solar cycles the expected ranges are expanded to 137-539 and 177-511 nT, which represent increases of $108 \%$ and $144 \%$ in the respective ranges. Thus there is at least a $99 \%$ probability that the daily a index will
\end{abstract}

\footnotetext{
*Also Visiting Reader in Physics, University of Sussex, Falmer, Brighton, BN1 9QH, UK

Correspondence to: D. M. Willis
}

satisfy the condition $a a<550$ for the largest geomagnetic storm in the next 100 solar cycles. The statistical analysis is used to infer that remarkable conjugate auroral observations on the night of 16 September 1770 , which were recorded during the first voyage of Captain Cook to Australia, occurred during an intense geomagnetic storm.

\section{Introduction}

Siscoe (1976) applied the statistics of extremes to the first, second and third largest geomagnetic storms in nine solar cycles (viz. 11 to 19), as measured by the average half-daily a index. His study is extended here to fourteen solar cycles (viz. 9 to 22) using the average daily $a a$ index for the interval 1844-1993. The conventional daily a index (Mayaud, 1980) is available electronically through the National Geophysical Data Center, Boulder, Colorado, for the interval 1868-1993, which extends by almost three solar cycles the time-interval considered by Siscoe. Moreover, the daily $a a$ index has recently been extended backwards in time by two solar cycles (1844-1868), using hourly measurements of magnetic declination made at the Helsinki Magnetic Observatory during the interval 1844-1880 (Nevanlinna and Kataja, 1993). Daily values of the "essentially equivalent" Helsinki magnetic activity index $A k$ are available electronically through the Finnish Meteorological Institute, Helsinki (Nevanlinna, 1995). Therefore, it is now possible to investigate the statistics of the first, second and third largest geomagnetic storms per solar cycle over a 150-year interval (1844-1993), using essentially homogeneous daily values of the $a a$ index of geomagnetic activity.

However, since the conventional daily $a a$ index is consistently homogeneous for only twelve solar cycles (viz. 11 to 22, as discussed in Sect. 2), the statistics of the largest geomagnetic storms per solar cycle are also 
studied for the shorter 126-year interval (1868-1993), as well as the 100-year interval (1868-1967) considered by Siscoe (1976). It is also important to consider all three cases separately because there are 137 missing daily values of the $A k$ index in the interval 1844-1867, which might just possibly influence the results for fourteen solar cycles. However, the majority of these missing values (83) lie in the interval 19 July 1856-9 October 1856, which is close to sunspot minimum (1856.0).

\section{Derivation of the $a a$ and $A k$ indices}

The $a$ index is derived from hand-scaled magnetograms from two almost antipodal observatories (at invariant magnetic latitudes of approximately $\pm 50^{\circ}$ ); one in the United Kingdom and the other in Australia (Mayaud, 1980; Menvielle and Berthelier, 1991). The subauroral observatories that have contributed to the derivation of the $a a$ index are Greenwich (1868-1925), Abinger (19261956) and Hartland (1957 - present) for the Northern Hemisphere; Melbourne (1868-1919), Toolangi (19201978) and Canberra (1979 - present) for the Southern Hemisphere.

For each 3-h interval (00:00-03:00, 03:00-06:00, etc.), $K$ indices (Mayaud, 1980; Menvielle and Berthelier, 1991; Joselyn, 1995) are derived for the two antipodal observatories. The $K$ index is a quasi-logarithmic number between 0 and 9 that is assigned to the end of these specified 3-h intervals. It is derived by measuring the maximum deviation [in nanoteslas (nT)] of the observed field from the expected quiet-time level, for each of the three magnetic-field components (Joselyn, 1995). The largest of the three maxima at each observatory is converted to a $K$ index by using a look-up table appropriate to that observatory. The $K$ indices measured at the two antipodal observatories are then converted back into amplitudes and an individual $a a$ index is the average of the two amplitudes, weighted to allow for the small difference in latitude of the northern and southern observatories, or for the slight changes in the locations of the two antipodal observatories.

Since an individual three-hourly value of the $a$ index is derived from just two $K$ indices, it provides only an approximate indication of the actual level of planetary geomagnetic activity. However, half-daily, or daily, averages of the $a a$ index give an acceptably accurate indication of geomagnetic activity on a global scale and over a significantly longer time-interval than any other index of geomagnetic activity (Menvielle and Berthelier, 1991). Mayaud (1973) published a unique 100-year (1868-1967) series of three-hourly $a a$ indices, which is based on $K$ indices that were all measured by the author himself to ensure the homogeneity of the time-series. The $a a$ indices have continued to be published subsequently, in order to provide a rapidly available worldwide index of geomagnetic activity that is physically meaningful on a half-daily or daily time-scale.

Nevanlinna and Kataja (1993) have essentially extended the $a a$ index backwards in time by more than two solar cycles (viz. 9 and 10); namely, from 1 July 1844 to
31 December 1867. Their daily index, designated $A k$, has been derived from hourly readings of declination $(D)$ made at the Helsinki Magnetic Observatory $\left(60^{\circ} 10.3^{\prime}\right.$ $\mathrm{N}, 24^{\circ} 59.0^{\prime} \mathrm{E}$ ) during the interval 1844-1880 (Nevanlinna et al., 1993; Nevanlinna and Ketola, 1993). Specifically, three-hourly $K$ indices and daily $A k$ amplitudes have been computed from declination values using an algorithm developed for the automatic production of $K$ indices in the case of modern digital data (Sucksdorff et al., 1991). To ensure that the Helsinki $K$ indices are as close as possible to the real ones, the percentage occurrence rate of $K$ values in each bin was adjusted to be the same as the corresponding distribution at the present-day Nurmijärvi Observatory, which is only $40 \mathrm{~km}$ from Helsinki. This was achieved by varying the Helsinki $K=9$ lower limit $\left(K_{9}\right)$; reasonably good agreement was found by fixing $K_{9}=200 \mathrm{nT}$. Then the three-hourly $K$ indices were converted into daily $A k$ amplitudes in the range $0-400 \mathrm{nT}$.

\section{Attributes and limitations of the extended $a a$ index}

The $a a$ index is used in the statistical study of the largest geomagnetic storms per solar cycle because it spans a much longer time-interval than any other index of geomagnetic activity (Mayaud, 1980; Menvielle and Berthelier, 1991). In addition, the aa index comprises a consistently uniform, homogeneous time-series, at least since 1868 , as required by the statistical analysis. For the period of overlap (1868-1880) between the conventional $a a$ index and the Helsinki $A k$ index, the linear relationship between the monthly means of the two indices is $a a=0.90( \pm 0.36)+1.07( \pm 0.02) \quad A k$, with a linear correlation coefficient of 0.96 (Nevanlinna and Kataja, 1993). Owing to the local-time character of magnetic disturbances at Helsinki, Nevanlinna and Kataja (1993) have expressed strong reservations about comparing the daily $a a$ and $A k$ indices. However, if days of missing $A k$ values are simply ignored, the corresponding linear relationship between the daily values of these indices is $a a=2.71( \pm 0.19)+0.93( \pm 0.01) A k$, with a linear correlation coefficient of 0.83 . This correlation is highly significant for 4415 daily values (there are 150 missing daily values of $A k$ in the interval 1 January 1868-30 June 1880), according to Student's $t$-test (Weatherburn, 1952). However, since the linear relationship between the daily values of $a a$ and $A k$ yields scaled ("corrected") values of $A k$ that are smaller than the original values if $A k>38.7$, the actual tabulated ("uncorrected") values of $A k$ are used in this study of extremes in geomagnetic activity.

Siscoe (1976) elected to use the half-daily a a index for two reasons: (1) it is referred to universal time rather than "storm time", which avoids any possible ambiguity relating to the actual definition of storm time; and (2) a time-scale of $12 \mathrm{~h}$ is intermediate between the substorm time-scale $(\sim 1 \mathrm{~h})$ and the storm time-scale $(\sim 24 \mathrm{~h})$. Hence the half-daily $a a$ index filters out substorm variations but retains the storm variation. The daily $a a$ index is used in this paper because half-daily values of 
the $A k$ index are not available; use of half-daily values of the $a a$ index would therefore eliminate solar cycles 9 and 10 from the present investigation. However, the statistical study of the first, second and third largest geomagnetic storms per solar cycle is undertaken separately for solar cycles 11 to 22 and for solar cycles 11 to 19 , again using the average daily a index. The latter set of results permits strict comparisons with the results derived by Siscoe (1976) using the half-daily aa index.

\section{Theory of the statistics of extremes}

As noted by Siscoe (1976), in every solar cycle there is a largest, second largest and third largest geomagnetic storm. The magnitudes of these three extreme storms in each solar cycle, as measured by the $a a$ index of geomagnetic activity, can be considered to be stochastic variables with some distribution of probabilities. In the theory of the statistics of extremes, it is shown that the probability distribution functions of extreme values have a common form for a wide range of phenomena (Fisher and Tippett, 1928; Gumbel, 1942, 1954, 1958; Court, 1952). This common form applies if the probability that a given observation is as large as a certain magnitude $x$, or larger than this magnitude, decreases asymptotically as $\exp (-x)$ or faster. In practice, it is not necessary to know the probability distribution for the entire set to verify that this condition holds. It suffices to confirm that the observed extremes comply with the form of the extreme-value distribution function appropriate to this condition (Gumbel, 1942, 1954). Following such confirmation, the free parameters in each distribution function can be found by a least-squares fit to the observations. This procedure yields analytic expressions for the probabilities of the first three extreme values as continuous functions of the magnitude $(x)$. These three analytic probability functions can be used to find the probabilities of extremes beyond the observed ranges.

If $\Phi_{m}(x)$ denotes the (asymptotic) probability that a given observation of an extreme $a a$ in a solar cycle is less than $x$, where $m=1,2$ and 3 for the first, second and third largest values, the theory of extremes gives for $\Phi_{m}(x)$ the following functional forms (Gumbel, 1954, 1958)

$$
\begin{aligned}
\Phi_{1}(x)= & \exp \left[-\exp \left(-y_{1}\right)\right], \\
\Phi_{2}(x)= & \exp \left[-2 \exp \left(-y_{2}\right)\right]\left[1+2 \exp \left(-y_{2}\right)\right], \\
\Phi_{3}(x)= & \exp \left[-3 \exp \left(-y_{3}\right)\right]\left[1+3 \exp \left(-y_{3}\right)\right. \\
& \left.+(9 / 2) \exp \left(-2 y_{3}\right)\right],
\end{aligned}
$$

where the reduced variate $y_{m}$ is defined by the linear relation

$y_{m}=a_{m} x+b_{m}$.

If the probability function for all $a a$ in a solar cycle were known, the constants $a_{m}$ and $b_{m}$ could be found directly from this function. Since it is not known, however, $a_{m}$ and $b_{m}$ must be found by fitting to the observations.

An important requirement in the theory of the statistics of extremes is that the extreme values should be independent. Therefore, no more than one extreme value is selected from any single storm period. In practice, this independence is achieved by ensuring that extreme values are separated in time by at least 30 days (i.e. by more than one synodic solar-rotation period). Following Siscoe (1976), it is assumed that any 80-year modulation of extreme-event amplitudes, produced by the approximately 80 -year period in solar activity, is sufficiently small to be neglected.

\section{Application of the statistics of extremes to geomagnetic storms}

Values of the daily $a a$ index for the first, second and third largest maxima in geomagnetic activity for solar cycles 9 to 22 are given in Table 1, together with their dates of occurrence. As noted previously, there are 137 missing daily values of the $a a(=A k)$ index during solar cycles 9 and 10 . The three maxima for solar cycle 22 are based on (final) daily values of the $a a$ index up to the end of 1993. However, the validity of these maxima has been checked by inspecting the tabular values of the
Table 1. Values and dates of the extreme daily $a a$ indices for the three largest geomagnetic storms of solar cycles 9-22 (for

\begin{tabular}{|c|c|c|c|c|c|c|}
\hline \multirow{2}{*}{$\begin{array}{l}\text { Solar-cycle } \\
\text { number }\end{array}$} & \multicolumn{2}{|c|}{ first maximum } & \multicolumn{2}{|c|}{ second maximum } & \multicolumn{2}{|c|}{ third maximum } \\
\hline & $a a(\mathrm{nT})$ & date & $a a(\mathrm{nT})$ & date & $a a(\mathrm{nT})$ & date \\
\hline $9 *$ & 178.0 & 20 Dec. 1847 & 160.0 & 24 Sep. 1847 & 148.0 & 20 Feb. 1852 \\
\hline $10^{*}$ & 159.0 & 9 Apr. 1858 & 141.0 & 19 Oct. 1865 & 139.0 & 17 Dec. 1857 \\
\hline 11 & 229.7 & 4 Feb. 1872 & 189.0 & 25 Oct. 1870 & 182.2 & 15 Oct. 1872 \\
\hline 12 & 304.6 & 20 Nov. 1882 & 292.2 & 17 Apr. 1882 & 154.2 & 30 Mar. 1886 \\
\hline 13 & 220.6 & 20 Jul. 1894 & 198.5 & 20 Aug. 1894 & 179.7 & 13 Feb. 1892 \\
\hline 14 & 329.0 & 25 Sep. 1909 & 308.1 & 31 Oct. 1903 & 144.3 & 29 Sep. 1908 \\
\hline 15 & 257.0 & 15 May 1921 & 196.3 & 17 Jun. 1915 & 180.1 & 11 Aug. 1919 \\
\hline 16 & 278.3 & 8 Jul. 1928 & 222.6 & 15 Oct. 1926 & 150.0 & 22 Jul. 1927 \\
\hline 17 & 349.5 & 18 Sep. 1941 & 302.0 & 5 Jul. 1941 & 249.5 & 1 Mar. 1941 \\
\hline 18 & 321.7 & 28 Mar. 1946 & 272.2 & 22 Sep. 1946 & 192.7 & 25 Jan. 1949 \\
\hline 19 & 352.1 & 13 Nov. 1960 & 346.6 & 15 Jul. 1959 & 304.7 & 8 Jul. 1958 \\
\hline 20 & 215.1 & 5 Aug. 1972 & 172.2 & 8 Mar. 1970 & 167.7 & 26 May 1967 \\
\hline 21 & 243.5 & 8 Feb. 1986 & 209.1 & 6 Sep. 1982 & 183.7 & 5 Feb. 1983 \\
\hline 22 & 348.2 & 13 Mar. 1989 & 212.8 & 5 Jun. 1991 & 189.3 & 10 May 1992 \\
\hline
\end{tabular}
solar-cycle numbers marked with *, the data are extracted from Nevanlinna (1995) 
(provisional) daily aa index published in the SolarGeophysical Data prompt reports (National Geophysical Data Center, Boulder, Colorado), to ensure that no larger values occurred in the interval 1 January 1994-30 April 1996. For solar cycles 11 to 19, all nine dates in Table 1 that specify the first maximum in the daily aa index (per solar cycle) are identical to the corresponding dates presented by Siscoe (1976, cf. his Table 1) for the half-daily $a a$ index. Similarly, seven of the dates of the second maximum are identical, whereas only two of the dates of the third maximum are identical.

According to both the "turning-point" and "phaselength" tests of randomness (Kendall and Stuart, 1976), each of the three sets of extreme values of the daily $a \mathrm{a}$ index presented in Table 1 is not significantly different from a random time-series if the hypothesis of randomness is tested at the level $P=0.01$ (the first and second maxima are not significantly different from random time-series if tested at the level $P=0.05)$. Empirical relationships between $\Phi_{m}(x)$ and $x(m=1,2,3)$ are derived by first arranging the three random sets of extreme $a a$ values in order of increasing magnitude, as shown in Table 2 for solar cycles 9 to 22. (Although not presented here, similar tables of ordered extreme aa values can be constructed for solar cycles 11 to 22 and 11 to 19.) Then the observed values of the probabilities $\Phi_{m}(x)$ are given by $\Phi_{m}(x)=[n(x)-1] / N$, where $n(x)$ is the ordinal number associated with each of the observed values of $x$ and $N$ is the total number of solar cycles. However, following the procedure recommended by Gumbel (1954) and Krumbein and Lieblein (1956), this definition of $\Phi_{m}(x)$ can be replaced by $\Phi_{m}(x)=$ $n(x) /(N+1)$. This procedure is useful for small samples because it enables all $N$ values to be used and it does not alter the results significantly. Thus Eq. 1 can be used to find the values of $y_{m}$ corresponding to each observed value of $x$, which can then be tested to see if they satisfy the linearity condition implied by Eq. 2 .

The results are shown in Fig. 1, where the respective symbols $\square, \triangle$ and $\diamond$ are used to denote points defining the first, second and third largest geomagnetic storms

Table 2. Extreme daily $a a$ indices from Table 1 arranged in order of increasing magnitude

\begin{tabular}{rlll}
\hline$n$ & $\begin{array}{l}\text { first maximum } \\
a a(\mathrm{nT})\end{array}$ & $\begin{array}{l}\text { second maximum } \\
a a(\mathrm{nT})\end{array}$ & $\begin{array}{l}\text { third maximum } \\
a a(\mathrm{nT})\end{array}$ \\
\hline 1 & 159.0 & 141.0 & 139.0 \\
2 & 178.0 & 160.0 & 144.3 \\
3 & 215.1 & 172.2 & 148.0 \\
4 & 220.6 & 189.0 & 150.0 \\
5 & 229.7 & 196.3 & 154.2 \\
6 & 243.5 & 198.5 & 167.7 \\
7 & 257.0 & 209.1 & 179.7 \\
8 & 278.3 & 212.8 & 180.1 \\
9 & 304.6 & 222.6 & 182.2 \\
10 & 321.7 & 272.2 & 183.7 \\
11 & 329.0 & 292.2 & 189.3 \\
12 & 348.2 & 302.0 & 249.5 \\
13 & 349.5 & 308.1 & 304.7 \\
14 & 352.1 & 346.6 & \\
\hline
\end{tabular}
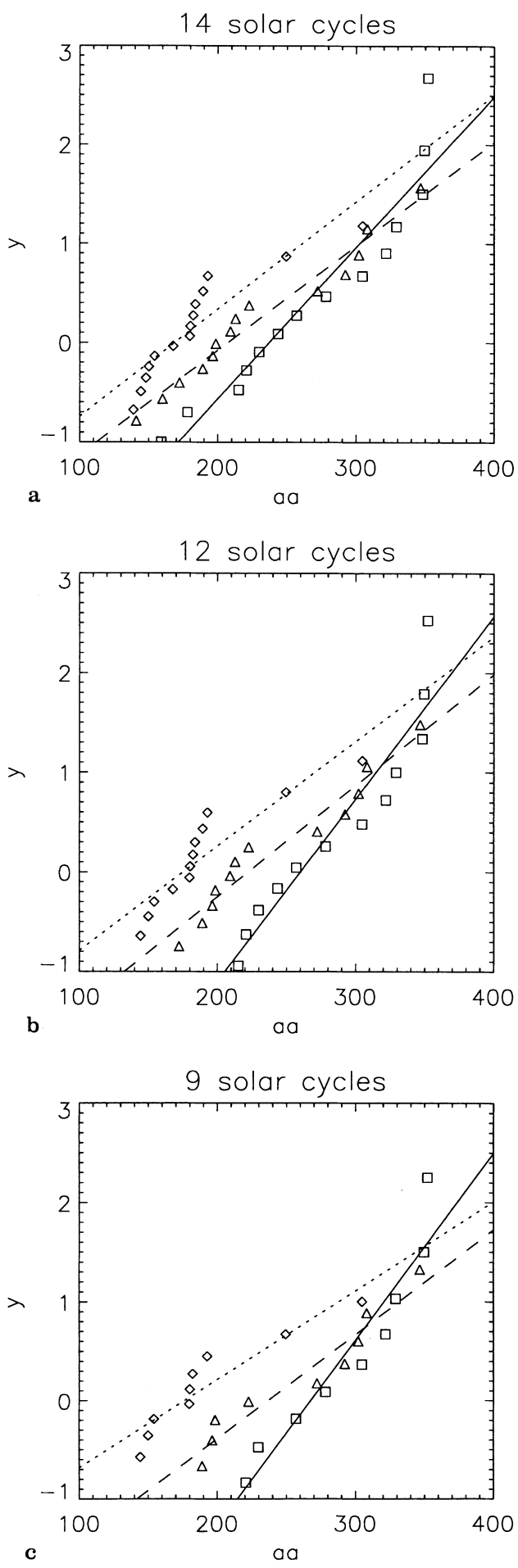

Fig. 1a-c. Observed extreme daily $a a$ indices for the first $(\square)$, second $(\triangle)$ and third $(\diamond)$ largest geomagnetic storms per solar cycle plotted against the "observed" values of $y$, as defined by Eq. 1: a solar cycles 9-22, b solar cycles 11-22 and c solar cycles 11-19. The straight lines are least-squares fits to the respective data points: the continuous, dashed and dotted lines refer to the first, second and third largest geomagnetic storms, respectively

per solar cycle. Parts a, b and c of the figure present results for solar cycles 9 to 22,11 to 22 and 11 to 19 , respectively. Straight-line fits to the data points by the method of least squares show that the linear relation- 
ships are quite well satisfied, at least for the first and second largest geomagnetic storms (i.e. $m=1$ and 2). By comparing Fig. 1c with the corresponding figure in the paper by Siscoe (1976), it is found that the linear relationship is slightly less well satisfied for the daily than the half-daily $a a$ index. The discrepancy between the daily and half-daily results for solar cycles 11 to 19 , measured in terms of the sums of squares of residuals, is most pronounced for the third largest geomagnetic storm $(m=3)$.

According to the theory of extremes, the probabilities of the three largest daily $a a$ indices per solar cycle are now determined by Eq. 1, together with the following specific linear relationships for solar cycles 9 to 22

$$
\begin{aligned}
& y_{1}=1.525(x-237.0) / 100, \\
& y_{2}=1.052(x-207.3) / 100, \\
& y_{3}=1.082(x-168.7) / 100 .
\end{aligned}
$$

The corresponding linear relationships for solar cycles 11 to 22 are

$$
\begin{aligned}
& y_{1}=1.831(x-259.9) / 100, \\
& y_{2}=1.117(x-222.2) / 100, \\
& y_{3}=1.050(x-175.1) / 100 .
\end{aligned}
$$

Finally, the linear relationships for just solar cycles 11 to 19 are

$$
\begin{aligned}
& y_{1}=1.887(x-267.6) / 100, \\
& y_{2}=1.055(x-236.7) / 100, \\
& y_{3}=0.897(x-176.2) / 100 .
\end{aligned}
$$

Table 3 presents the observed means and $( \pm)$ the observed standard deviations for the first, second and third largest geomagnetic storms in solar cycles 9 to 22 (cf. Table 2), 11 to 22 and 11 to 19. Figure 1 and Table 3 permit comparisons to be made between the different results obtained for solar cycles 9 to 22,11 to 22 and 11

Table 3. Observed means and standard deviations of the $a a$ index for the three largest geomagnetic storms of solar cycles 9 to 22,11 to 22 and 11 to 19

\begin{tabular}{rlll}
\hline $\begin{array}{l}\text { Solar-cycle } \\
\text { numbers }\end{array}$ & $\begin{array}{l}\text { first maximum } \\
\text { a }(\mathrm{nT})\end{array}$ & $\begin{array}{l}\text { second maximum } \\
\text { a }(\mathrm{nT})\end{array}$ & $\begin{array}{l}\text { third maximum } \\
\text { a }(\mathrm{nT})\end{array}$ \\
\hline 9 to 22 & $270.5 \pm 65.2$ & $230.2 \pm 62.8$ & $183.2 \pm 44.9$ \\
11 to 22 & $287.4 \pm 53.0$ & $243.5 \pm 57.4$ & $189.8 \pm 45.2$ \\
11 to 19 & $293.6 \pm 49.6$ & $258.6 \pm 58.1$ & $193.0 \pm 52.3$ \\
\hline
\end{tabular}

Table 4. Statistical characteristics of the three largest daily $a a$ indices per solar cycle (in nanoteslas) for solar cycles 9 to 22. Pairs of numbers in parentheses are the corresponding values for solar to 19. It should be emphasized again that results for solar cycles 11 to 22 are based entirely on the use of the consistently homogeneous aa index, and extend the results obtained by Siscoe (1976) for solar cycles 11 to 19 by including the three most recent solar cycles. Results for solar cycles 9 to 22 involve the inclusion of two additional solar cycles prior to 1868 , which requires the use of the Helsinki $A k$ index. Although the daily $A k$ index is intended to provide an extension of the daily $a a$ index backwards in time to 1844 (Nevanlinna and Kataja, 1993), it may not provide a completely homogeneous extension of this latter index.

Visual inspection of the statistical data presented in Fig. 1 and Table 3 suggests that the results for solar cycles 11 to 22 are essentially intermediate between those for solar cycles 9 to 22 and 11 to 19 . However, it appears that the noticeably smaller values of the $a a$ index for the largest (and possibly second largest) geomagnetic storms in solar cycles 9 and 10 (cf. Table 1) may be exerting a significant influence on the statistical results. For this reason, all subsequent statistical calculations are performed separately for solar cycles 9 to 22,11 to 22 and 11 to 19 .

\section{Statistical characteristics of the extreme values}

The standard statistical parameters of the reduced variate $y_{m}(m=1,2,3)$, namely the mode, median, mean and standard deviation can be determined from the distribution functions (Gumbel, 1958). Using these standard values of $y_{m}$, the associated values of $x$ can be derived from Eqs. 3, 4 and 5. Numerical values of these standard statistical parameters for the daily $a a$ index are presented in Table 4 for solar cycles 9 to 22 . Pairs of numbers in parentheses are the corresponding numerical values for solar cycles 11 to 22 (first number) and 11 to 19 (second number): this abbreviation is adopted throughout the remainder of the paper. Each second value in parentheses can be compared directly with the equivalent numerical value for the half-daily $a a$ index presented in Table 3 of the paper by Siscoe (1976). The numbers in Table 4 indicate that the most probable and average values of the daily $a a$ index for the three largest storms are well separated. This statement is true for the full (9 to 22) and restricted (both 11 to 22 and 11 to 19) number of solar cycles, and is in agreement with the conclusion reached by Siscoe (1976) for the halfdaily $a a$ index.

cycles 11 to 22 (first number) and 11-19 (second number). (Relative dispersion derived by dividing the standard deviation by the mode.)

\begin{tabular}{llllr}
\hline$m$ & mode & median & mean & $\begin{array}{c}\text { standard } \\
\text { deviation } \\
\text { dispersion }\end{array}$ \\
\hline 1 & $237.0(259.9,267.6)$ & $261.0(279.9,287.0)$ & $274.9(291.4,298.2)$ & $84.1(70.0,68.0)$ \\
2 & $207.3(222.2,236.7)$ & $224.0(237.9,253.3)$ & $233.0(246.4,262.3)$ & $0.35(0.27,0.25)$ \\
3 & $168.7(175.1,176.2)$ & $179.3(186.1,189.0)$ & $185.0(191.8,195.8)$ & $58.1(59.9,76.1)$ \\
\hline
\end{tabular}


However, the relative dispersion $(=$ standard deviation /mode) of the daily a index is essentially independent of $m$ for solar cycles 9 to 22 , whereas it increases significantly as $m$ increases from 1 to 3 for solar cycles 11 to 19 , as in the case of the half-daily $a a$ index. For solar cycles 11 to 22 , the relative dispersion increases significantly as $m$ increases from 1 to 2 but then only increases slightly as $m$ increases from 2 to 3 . These results suggest that, as the number of solar cycles increases from 9 to 14 , the relative dispersion tends to an almost constant value, at least for $m=1$ to 3 . The relative dispersion is important because it defines the number of solar cycles required to have an appreciable probability that the extreme value is very far from the modal value. If the relative dispersion is comparatively small, a larger number of solar cycles is required. Conversely, if the relative dispersion is comparatively large, a smaller number of solar cycles suffices.

The mean and standard deviation in Table 4 are for an infinite number of solar cycles; the expected mean and standard deviation are smaller for a finite number of cycles. For example, in the case $m=1$ for $14(12,9)$ cycles, the mean is $270.4(287.4,293.6)$ and the standard deviation is $66.2(53.7,49.2)$. These expected values for $14(12,9)$ solar cycles are in remarkably good agreement with the observed values presented in Table 3 , namely $270.5(287.4,293.6)$ and $65.2(53.0,49.6)$.

\section{Probabilities of the three largest geomagnetic storms per solar cycle}

Equations 1-5 can be used to determine the (asymptotic) probabilities of the three largest geomagnetic storms per solar cycle as functions of the $a a$ index. Figure $2 \mathrm{a}-\mathrm{c}$ shows plots of the probability $\Phi_{m}(x)(m=1,2$ and 3$)$ that a given observation of an extreme geomagnetic storm in a solar cycle is less than $a a$. The continuous, dashed and dotted curves refer to the first $(m=1)$, second $(m=2)$ and third $(m=3)$ largest geomagnetic storms. Following the convention adopted for Fig. 1, a, b and c of Fig. 2 refer, respectively, to solar cycles 9-22, 11-22 and 11-19.

For the first largest geomagnetic storms $(m=1)$, the continuous curves in $\mathrm{b}$ and $\mathrm{c}$ of Fig. 2 show a very similar variation of $\Phi(x)$ with increasing $a a$. Conversely, the continuous curve in Fig. $2 \mathrm{a}$ is significantly different to the continuous curves in $\mathrm{b}$ and $\mathrm{c}$, particularly for the lower $a a$ values $(a a<300)$. This discrepancy almost certainly arises from the noticeably lower observed values of the first largest geomagnetic storms for solar cycles 9 and 10 (cf. Table 1). For the second largest geomagnetic storms $(m=2)$, the dashed curves in Fig. $2 \mathrm{a}-\mathrm{c}$ exhibit a rather more gradual change in the variation of $\Phi(x)$ with $a a$ as the number of solar cycles increases from 9 to 14. Finally, for the third largest geomagnetic storms $(m=3)$, the dotted curves in Fig. $2 \mathrm{a}-\mathrm{c}$ reveal only minor differences in the variation of $\Phi(x)$ with $a a$ as the number of solar cycles increases from 9 to 14 . Notwithstanding the detailed differences between the comparable curves presented in Fig. $2 \mathrm{a}-\mathrm{c}$,
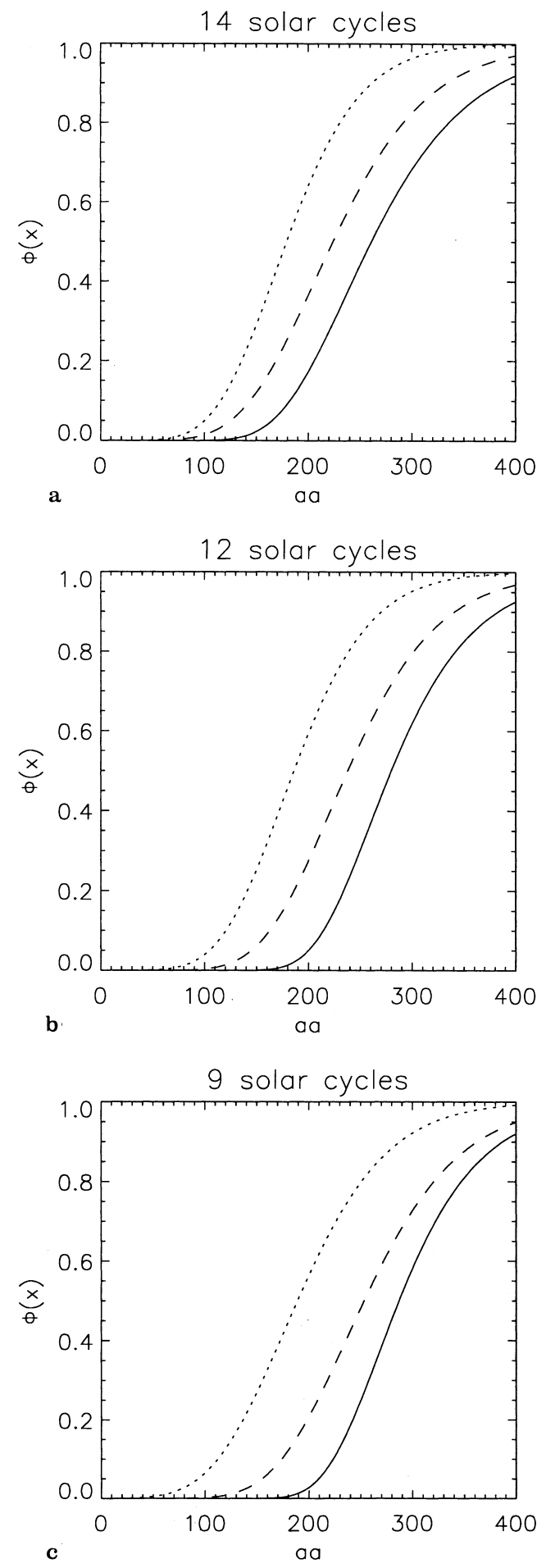

Fig. 2a-c. Plots of the probability that a given observation of a first, second and third largest geomagnetic storm $(m=1,2$ and 3$)$ in a solar cycle is less than $a a$ : a solar cycles 9-22, b solar cycles 11-22 and c solar cycles 11-19. The continuous, dashed and dotted curves refer to the first $(m=1)$, second $(m=2)$ and third $(m=3)$ largest geomagnetic storms, respectively

the general trend of the variation of $\Phi_{m}(x)(m=1,2$ and 3 ) with increasing $a a$ does not depend critically on the number of solar cycles included in the statistical analysis. 


\section{The expected ranges of extreme storms for a given number of solar cycles}

The probabilities presented in Fig. 2 can be used to calculate the expected ranges of the first, second and third largest geomagnetic storms ( $m=1,2$ and 3 ) for a given number of solar cycles. The growth in the expected ranges of extreme values of the daily $a a$ index as a function of the number of cycles can be estimated from the equations $T_{1}(x)=[1-\Phi(x)]^{-1}$, the expected number of cycles required to have one cycle with an extreme equal to or greater than $x(=a a)$, and $T_{2}(x)=[\Phi(x)]^{-1}$, the expected number of cycles required to have one cycle with an extreme less than $x$ (Gumbel, 1958). This procedure is strictly valid, of course, only if the number of cycles is large enough to eliminate the possibility of a biased sample. The functions $T_{1}(x)$ and $T_{2}(x)$ are plotted in Fig. 3 for the three largest extremes $(m=1,2,3)$ : Fig. 3 a refers to solar cycles 9 to 22 , Fig. $3 b$ refers to solar cycles 11 to 22 and Fig. 3c refers to solar cycles 11 to 19 . In each case the curves labelled $T_{1}$ and $T_{2}$ meet at the point where $T_{1}=T_{2}=2$, which is located at the median value of the extreme daily $a a$ index.

The continuous, dashed and dotted curves in Fig. 3a can be interpreted as implying that for 14 solar cycles (9 to 22) all but two of the observed extreme values of the daily $a a$ index are expected to be confined to the ranges $173.4<(a a)_{1}<407.6,134.4<(a a)_{2}<352.1$ and $107.7<(a a)_{3}<274.9$, where the subscripts denote values of $m$. For each of the three ranges $(m=1,2,3)$, one observed extreme is expected to lie outside the range to the right and one outside to the left. This theoretical expectation is not realized precisely for the observed extremes presented in Table 2 because these extremes do not lie exactly on the least-squares fits to the data points, which are used to derive Fig. 3a, as is clear from Fig. 1a. As the number of cycles increases, however, the ranges indicated in Fig. 3a become increasingly better representations of the true expected ranges. For a group of 100 solar cycles, the corresponding statistical ranges for the continuous, dashed and dotted curves are $136.9<(a a)_{1}<538.6,93.3<(a a)_{2}<454.4$ and $73.5<$ $(a a)_{3}<346.9$. Similarly, the equivalent statistical ranges for observations over 12 solar cycles (11 to 22) are $176.5<(a a)_{1}<511.1,114.8<(a a)_{2}<455.0$ and $77.0<(a a)_{3}<358.8$ (cf. Fig. 3b); the equivalent statistical ranges for observations over 9 solar cycles are $186.7<(a a)_{1}<511.4,123.0<(a a)_{2}<483.1$ and $61.3<(a a)_{3}<391.2$ (cf. Fig. 3c).

If the expected dilatation for a given number of cycles is measured by the ratio of the largest to the smallest value in the range, then for 100 cycles the ratios are 3.9 for $(a a)_{1}, 4.9$ for $(a a)_{2}$ and 4.7 for $(a a)_{3}$. These ranges and dilatations are based on measured values of the daily $a a$ index for solar cycles 9 to 22 . The corresponding dilatations based on measured values of the daily $a a$ index for solar cycles 11 to 22 are 2.9, 4.0 and 4.7. Similarly, the dilatations based on measured values of the daily $a a$ index for just solar cycles 11 to 19 are 2.7 , 3.9 and 6.4. These latter three values can be compared
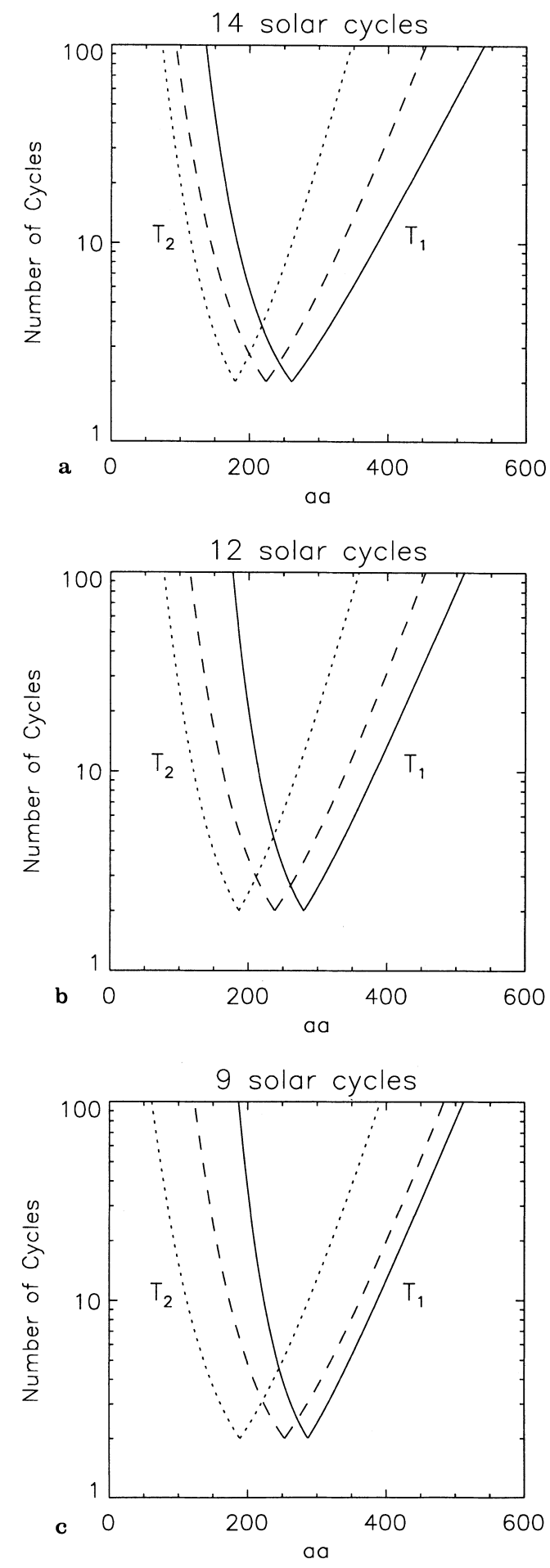

Fig. 3a-c. Curves defining the expected number of solar cycles required to find one cycle with an extreme value of $a a$ equal to or greater than the abscissa ( $T_{1}$ branch) and to find one cycle with an extreme value of $a a$ less than the abscissa $\left(T_{2}\right.$ branch) for the three largest $(m=1,2$ and 3$)$ geomagnetic storms per solar cycle: a solar cycles 9-22, b solar cycles 11-22 and c solar cycles 11-19. The continuous, dashed and dotted curves refer to the first $(m=1)$, second $(m=2)$ and third $(m=3)$ largest geomagnetic storms, respectively

directly with the equivalent values derived implicitly by Siscoe (1976) for the half-daily $a a$ index, namely 2.2, 3.4 and 7.3. Therefore, the expected dilatations of both the 
daily and half-daily $a a$ indices for solar cycles 11 to 19 vary significantly as $m$ increases from 1 to 3 . Conversely, the variation of the expected dilatations of the daily $a$ a index as $m$ increases is appreciably less for all fourteen solar cycles (9 to 22) than for the subset of nine solar cycles (11 to 19). An intermediate situation prevails for twelve solar cycles (11 to 22), although the behaviour as $m$ increases is probably closer to that for nine solar cycles (11 to 19 ). This last result again suggests that the noticeably smaller values of the $a a$ index for the largest (and possibly second largest) geomagnetic storms in solar cycles 9 and 10 may be exerting a significant influence on the statistical results.

More generally, the curves in Fig. 3a, which are based on the extreme values of the daily $a a$ index for solar cycles 9 to 22, indicate that the expected range decreases significantly as $m$ increases from 1 to 3 . The curves in Fig. 3b, which are based on the extreme values of the daily $a a$ index for solar cycles 11 to 22 , indicate that the expected range again decreases significantly as $m$ increases from 1 to 3 , although there is a very small increase as $m$ increases from 1 to 2. Conversely, the curves in Fig. 3c, which are based on the extreme values of the daily $a a$ index for solar cycles 11 to 19 , indicate that the expected range varies less as $m$ increases but is again greatest for $m=2$. Therefore, for the interval 1844-1993 (i.e. solar cycles 9 to 22), the expected ranges of the daily $a a$ index for the first, second and third largest geomagnetic storms per solar cycle decrease monotonically in magnitude. This result is the exact opposite of the one found by Siscoe (1976) for the expected ranges of the half-daily aa index during the shorter interval 1868-1967 (i.e. for solar cycles 11 to 19). The expected ranges of the daily aa index for this shorter interval exhibit a more neutral behaviour.

The statistical procedures discussed in this section provide only a rather limited opportunity of testing the predictive accuracy of extreme-value statistics, as applied to the three largest geomagnetic storms per solar cycle. Based on observations of the three largest values of the daily $a a$ index per solar cycle for the 100-year interval 1868-1967 (i.e. solar cycles 11-19; cf. Table 1), Eqs. 1 and 5 can be used to estimate the expected ranges for 12 and 14 solar cycles (cf. Fig. 3c) The theoretical expected ranges for the first, second and third largest geomagnetic storms per solar cycle are $219.4<(a a)_{1}<397.0$, $168.3<(a a)_{2}<372.6$ and $106.9<(a a)_{3}<296.9$, for 12 solar cycles; and $216.2<(a a)_{1}<405.5,164.0<$ $(a a)_{2}<381.1$ and $102.7<(a a)_{3}<304.3$, for 14 solar cycles. In the case of the largest geomagnetic storms [viz. $(a a)_{1}$ ] for solar cycles 11-22, one observed extreme (5 August 1972) is less than 219.4, as required by the theory, but no observed extreme is greater than 397.0. In the case of the largest geomagnetic storms for solar cycles 9-22, three observed extremes (20 December 1847, 9 April 1858 and 5 August 1972) are less than 216.2 and no observed extreme is greater than 405.5 This last result suggests once again that the noticeably smaller values of the $a a$ index for the first largest geomagnetic storms in solar cycles 9 and 10 may be exerting a significant influence on the statistical results.
As noted previously, however, the theoretical expectation is not realized in practice even for the observed extremes of solar cycles 11 to 19 (cf. Table 1) because these extremes do not lie exactly on the least-squares fits to the data points, as is clear from Fig. 1c. Moreover, considerable circumspection must be exercised in any attempt to use the statistics of extremes to predict the largest geomagnetic storms in the next solar cycle. The great merit of the theory of extremes is that it enables increasingly better estimates to be made of the true expected ranges as the time-interval for the prediction becomes increasingly long compared with the timeinterval for which observations exist. On the basis of the results presented in this paper, it can be claimed with confidence that there is at least a $99 \%$ probability that the daily a $a$ index satisfies the condition $a a<550$ for the largest geomagnetic storm in 100 solar cycles, as implied by Fig. 3a-c. The corresponding condition for the largest geomagnetic storm in 500 solar cycles (i.e. approximately five-and-a-half millennia) is $a a<650$.

\section{Conclusions and discussion}

A previous study of the largest geomagnetic storms per solar cycle for the 100-year interval 1868-1967 (Siscoe, 1976) is extended to the 150-year interval 1844-1993. The intensity of a geomagnetic storm is classified in this paper by the magnitude of the daily $a a$ index rather than the half-daily $a a$ index. The latter index was preferred by Siscoe (1976) because a time-scale of $12 \mathrm{~h}$ is intermediate between the substorm time-scale $(\sim 1 \mathrm{~h})$ and the storm time-scale $(\sim 24 \mathrm{~h})$. However, inclusion of the essentially equivalent Helsinki magnetic activity index $A k$ (Nevanlinna and Kataja, 1993), which is available only on a daily basis for the interval 1844-1868, inevitably restricts the present study to the daily a index of magnetic activity. Since the $A k$ index may not provide an entirely homogeneous extension of the $a a$ index, the statistical analysis is performed separately for twelve solar cycles (1868-1993), as well as nine solar cycles (1868-1967).

Many other studies of geomagnetic storms and magnetic activity have been undertaken using different magnetic indices and classification criteria (e.g. Gosling et al., 1991; Tsurutani et al., 1992; Taylor et al., 1994; Silbergleit, 1996, 1997). However, these studies usually relate geomagnetic storms to satellite measurements of various physical properties of the solar wind and interplanetary magnetic field. Such studies are necessarily restricted to just the last few solar cycles, whereas the present investigation uses the only magnetic index $(a a)$ that spans 14 solar cycles.

An important conclusion of the application of extreme-value statistics to the daily $a a$ index for the longer interval (1844-1993) is that the relative dispersion (= standard deviation/mode) does not vary significantly between the first $(m=1)$, second $(m=2)$ and third $(m=3)$ largest geomagnetic storms per solar cycle (cf. Table 4). Over the shorter time-interval (1868-1967), however, the relative dispersion varies significantly (viz. increases monotonically as $m$ increases) for both the 
daily and half-daily $a a$ indices. Conversely, for the longer interval (1844-1993), the expected ranges of the daily $a a$ index for the first, second and third largest geomagnetic storms per solar cycle decrease monotonically in magnitude as $m$ increases (cf. Fig. 3a). This latter result is the exact opposite of the result derived by Siscoe (1976) for the shorter interval (1868-1967), during which the corresponding expected ranges of the half-daily $a a$ index increase monotonically in magnitude as $m$ increases. The expected ranges of the daily $a a$ index for this shorter interval (1868-1967) exhibit a more neutral behaviour; indeed, the largest expected range occurs for $m=2$, as it does in the case of the intermediate interval (1868-1993).

The statistical results derived in this paper can be used to place an upper limit on the largest geomagnetic storm that is likely to occur in a much longer period of time, which may be imagined to extend either backwards into the past or forwards into the future. For example, there is at least a $99 \%$ probability that the daily a $a$ index satisfies the condition $a a<550$ for the largest geomagnetic storm in 100 solar cycles. The corresponding condition for 500 solar cycles (about 5500 years) is $a a<650$. Such predictions of the largest geomagnetic storm that might occur in the future may be of some value to experimentalists designing sensitive instruments to be flown on spacecraft exploring the near-Earth environment. Similar predictions can be made for the lower limit of the largest geomagnetic storm in 100 (or 500) solar cycles. Siscoe (1976) used a lower limit appropriate to the half-daily $a a$ index to infer that it is unlikely that any solar cycle in recent history would have passed without the occurrence of at least one geomagnetic storm that produced auroral displays visible at mid-latitudes, provided the statistics (for the longer interval) were the same as those for nine solar cycles (viz. 11 to 19). An analogous argument is adduced at the end of this section in a discussion of the remarkable conjugate auroral observations during the night of 16 September 1770 (Willis et al., 1996).

By comparing the results obtained in this paper with those presented by Siscoe (1976), it is clear that results derived by applying the statistics of extremes to the first, second and third largest geomagnetic storms per solar cycle depend on the actual time-interval considered. The results also depend, though to a lesser extent, on whether the daily or half-daily $a a$ index is used as a measure of the intensity of a geomagnetic storm. Both these dependencies are to be expected for a statistical analysis based on a relatively small number of solar cycles. Nevertheless, the main scientific conclusions reached by Siscoe (1976) are confirmed by the present investigation. In particular, it remains unlikely that any solar cycle in recent history would have passed without one geomagnetic storm that produced auroral displays visible at mid-latitudes, provided the statistics were the same as those for the last 14 solar cycles.

In this context, it is instructive to apply the preceding statistical analysis to observations of the aurora australis on the night of 16 September 1770, recorded by Banks (Beaglehole, 1962) and Parkinson (1773) on board
HMS Endeavour during the first voyage of Captain James Cook to Australia (Eather, 1980). Both recorded descriptions of the aurora australis refer to a red light or glow in the southern sky accompanied by rays, or stripes, of a brighter coloured light extending directly upwards. This auroral display was observed at around 23:00 LT, at which time HMS Endeavour was about $10^{\circ} \mathrm{S}$ of the equator between Timor and the island of Savu. Observations of the aurora borealis on the same night, and the two following nights, have been recorded in Chinese provincial histories, which refer to auroral displays seen in the northern sky from several sites in China about $40^{\circ} \mathrm{N}$ of the equator (Willis et al., 1996). These observations provide the earliest example yet known of conjugate auroral sightings and they probably occurred during an intense geomagnetic storm.

According to the statistical analysis given here, $164<(a a)_{1}<435$ for a group of 21 solar cycles (cf. Fig. 3a), which corresponds to (backwards) extrapolation over the time-interval 1767-1993 (McKinnon, 1987). Following the reasoning adduced by Siscoe (1976), $a a=164$ is taken to represent the expected minimum largest geomagnetic storm for 21 cycles. This particular value of $a a$ is almost equal to the values for the geomagnetic storms of 24 September 1847, 9 April 1858 and 26 May 1967 (cf. Table 1). As noted in the catalogue published by Angot (1896), the storm of 24 September 1847 produced an aurora that was visible from Greenwich $\left(51.29^{\circ} \mathrm{N}, 0.00^{\circ} \mathrm{E}\right)$, while the storm of 9 April 1858 produced an aurora that was visible from Senftenberg $\left(51.31^{\circ} \mathrm{N}, 14.01^{\circ} \mathrm{E}\right.$ ) and Breslau (Wrocław; $51.05^{\circ} \mathrm{N}, 17.00^{\circ} \mathrm{E}$ ), amongst other European cities. The more recent storm of 26 May 1967, which is understandably much better documented (e.g. Findlay et al., 1969), produced an aurora that was visible from many locations in the United States of America and Canada. One observation from as far south as Alabama $\left(\approx 35^{\circ} \mathrm{N}\right)$, and several nearly overhead observations from Washington $\mathrm{DC}\left(38.55^{\circ} \mathrm{N}, 77.00^{\circ} \mathrm{W}\right)$, were reported. Therefore, since there is a clear correspondence between the latitude of overhead auroral displays and the magnitude of the aa index (Legrand and Simon, 1989), it seems highly likely that the remarkable conjugate auroral observations on the night of 16 September 1770 (Willis et al., 1996) were associated with an intense geomagnetic storm. This conclusion is corroborated by the fact that auroral displays were also observed from several places in both Europe (Angot, 1896) and Japan (Matsushita, 1956; Willis et al., 1996) on the following night.

Acknowledgements. The authors thank Mr M. N. Wild (World Data Centre C1 for Solar-Terrestrial Physics, Rutherford Appleton Laboratory) for obtaining the daily values of the $a a$ index from the National Geophysical Data Center, Boulder, Colorado. They also thank Mrs J. Foster for help with the preparation of the paper. The research reported was initiated while PRS worked at the Rutherford Appleton Laboratory for a month as a British Physics Olympiad Vacation Student; his present address is: Clare College, University of Cambridge, Cambridge CB2 1TL, UK. Constructive comments by two referees have resulted in substantial improvements to the paper. 
Topical Editor K.-H. Glaßmeier thanks M. Lester and R. Friedel for their help in evaluating this paper.

\section{References}

Angot, A., The aurora borealis, Kegan Paul, Trench, Trübner and Co., London, 1896.

Beaglehole, J. C., The endeavour journal of Joseph Banks 17681771, Vol. II, Angus and Robertson, Sydney, 1962.

Court, A., Some new statistical techniques in geophysics, in Advances in geophysics, Vol. 1, Ed. H. E. Landsberg, Academic Press, New York, pp. 45-85, 1952.

Eather, R. H., Majestic lights: the aurora in science, history, and the arts, American Geophysical Union, Washington DC, 1980.

Findlay, J. A., P. L. Dyson, L. H. Brace, A. J. Zmuda, and W. E. Radford, Ionospheric and magnetic observations at $1000 \mathrm{~km}$ during the geomagnetic storm and aurora of 25-26 May 1967, J. Geophys. Res., 74, 3705-3712, 1969.

Fisher, R. A., and L. H. C. Tippett, Limiting forms of the frequency distribution of the largest or smallest member of a sample, Proc. Camb. Phil. Soc., 24, 180-190, 1928.

Gosling, J. T., D. J. McComas, J. L. Phillips, and S. J. Bame, Geomagnetic activity associated with Earth passage of interplanetary shock disturbances and coronal mass ejections, $J$. Geophys. Res., 96, 7831-7839, 1991.

Gumbel, E. J., On the frequency distribution of extreme values in meteorological data, Bull. Am. Meteorol. Soc., 23, 95-105, 1942.

Gumbel E. J., Statistical theory of extreme values and some practical applications, Appl. Math. Ser., Vol. 33, National Bureau of Standards, Washington DC, 1954.

Gumbel E. J., Statistics of extremes, Columbia University Press, New York, 1958.

Joselyn, J. A., Geomagnetic activity forecasting: the state of the art, Rev. Geophys., 33, 383-401, 1995.

Kendall, M. G., and A. Stuart, The advanced theory of statistics, Vol. 3 (3rd Ed.), Charles Griffin, London, High Wycombe, 1976.

Krumbein, W. C., and J. Lieblein, Geological application of extreme-value methods to interpretation of cobbles and boulders in gravel deposits, EOS, Trans. Am. Geophys. Union, 37, 313-319, 1956.

Legrand, J.-P., and P. A. Simon, Solar cycle and geomagnetic activity: a review for geophysicists. Part I. The contributions to geomagnetic activity of shock waves and of the solar wind, Ann. Geophysicae, 7, 565-578, 1989.

Matsushita, S., Ancient aurorae see in Japan, J. Geophys. Res., 61, 297-302, 1956.

Mayaud, P. N., A hundred-year series of geomagnetic data, 18681967, indices $a a$, storm sudden comencements, IAGA Bull. 33, IUGG Publication Office, Paris, 1973.
Mayaud, P. N., Derivation, meaning, and use of geomagnetic indices, Geophys. Monogr. 22, American Geophysical Union, Washington DC, 1980.

McKinnon, J. A., Sunspot numbers: 1610-1985. Report UAG-95, World Data Center A for Solar-Terrestrial Physics, Boulder, Colorado, 1987.

Menvielle, M., and A. Berthelier, The $K$-derived planetary indices: description and availability, Rev. Geophys., 29, 415-432, 1991.

Nevanlinna, H., New geomagnetic activity index series published for 1844-1880, EOS, Trans. Am. Geophys. Union, 76, 233-234, 1995.

Nevanlinna, H., and E. Kataja, An extension of the geomagnetic activity index series $a a$ for two solar cycles (1844-1868), Geophys. Res. Lett., 20, 2703-2706, 1993.

Nevanlinna, H., and A. Ketola, Magnetic results from Helsinki magnetic-meteorological observatory. Part III: Declination 1854-1880. Geomagnetic activity 1884-1880, Finn. Met.-Inst.Geophys. Publ., 32, 1993.

Nevanlinna, H., A. Ketola, L. Häkkinen, A. Viljanen, and K. Ivory, Geomagnetic activity during solar cycle 9 (1844-1856), Geophys. Res. Lett., 20, 743-746, 1993.

Parkinson, S., A journal of a voyage to the South Seas in his Majesty's Ship, The Endeavour. Faithfully transcribed from the Papers of the late Sydney Parkinson, Draughtsman to Joseph Banks, Esquire, on his late Expedition, with Dr Solander, round the World. Embellished with views and designs, delineated by the Author, and engraved by capital Artists, Printed for Stanfield Parkinson, the Editor, and sold by Messrs Richardson and Urquhart, at the Royal Exchange, [and others] London, 1773. Facsimile edition, 1972.

Silbergleit, V. M., On the occurrence of geomagnetic storms with sudden commencements, J. Geomagn. Geoelectr., 48, 10111016, 1996.

Silbergleit, V.M., On the occurrence of the largest geomagnetic storms per solar cycle, J. Atmos. Ten. Phys., 59, 259-262, 1997.

Siscoe, G. L., On the statistics of the largest geomagnetic storms per solar cycle, J. Geophys. Res., 81, 4782-4784, 1976.

Sucksdorff, C., R. Pirjola, and L. Häkkinen, Computer production of $K$-indices based on linear elimination, Geophys. Trans., 36, 333-345, 1991.

Taylor, J. R., M. Lester, and T. K. Yeoman, A superposed epoch analysis of geomagnetic storms, Ann. Geophysicae, 12, 612-624, 1994.

Tsurutani, B. T., W. D. Gonzalez, F. Tang, and Y. T. Lee, Great magnetic storms, Geophys. Res Lett., 19, 73-76, 1992.

Weatherburn, C. E., A first course in mathematical statistics, Cambridge University Press, London, 1952.

Willis, D. M., F. R. Stephenson, and J. R. Singh, Auroral observations on A.D. 1770 September 16: the earliest known conjugate sightings, Q. J. R. Astron. Soc., 37, 733-742, 1996. 